Article

\title{
Boosting Second Harmonic Radiation from AlGaAs Nanoantennas with Epsilon-Near-Zero Materials
}

\author{
Davide Rocco $^{1, *(\mathbb{D})}$, Maria Antonietta Vincenti ${ }^{1}(\mathbb{D})$ and Costantino De Angelis ${ }^{1,2}$ (D) \\ 1 Department of Information Engineering, University of Brescia, Via Branze 38, 25123 Brescia, Italy; \\ maria.vincenti@unibs.it (M.A.V.); costantino.deangelis@unibs.it (C.D.A.) \\ 2 National Institute of Optics (INO), Via Branze 45, 25123 Brescia, Italy \\ * Correspondence: d.rocco003@unibs.it; Tel.: +39-030-371-5934
}

Received: 28 September 2018; Accepted: 7 November 2018; Published: 10 November 2018

\begin{abstract}
Enhancing the second harmonic conversion efficiency at the nanoscale is a critical challenge in nonlinear optics. Here we propose the use of epsilon-near-zero materials to boost the nonlinear radiation in the far field. Here, a comparison of the second harmonic behavior of a cylindrical AlGaAs nanoantenna placed over different semi-infinite layers is presented. In particular, we observed that the second harmonic generation is strongly enhanced and redirected by the simultaneous presence of a resonance at the fundamental wavelength and a low-permittivity condition in the substrate at the harmonic wavelength. Our results pave the way for a novel approach to enhance optical nonlinearities at the nanoscale.
\end{abstract}

Keywords: optical nanoantennas; linear and nonlinear nanoantennas; epsilon-near-zero materials

\section{Introduction}

Dielectric nanoantennas have recently gained increasing interest for many applications such as directional scattering and emission, nonlinear spectroscopy and microscopy [1-4]. Recently, a nanoscale system based on $\mathrm{AlGaAs}$ nanodisks placed over an $\mathrm{AlO}_{\mathrm{x}}$ substrate pumped in the telecom range was theoretically proposed as an efficient system to enhance second-order nonlinear effects in nanoscale optics [5]. The conversion efficiencies reported were higher than $10^{-5}$ [6-8], which is more than four orders of magnitude greater than optimized centrosymmetric plasmonic nanoantennas pumped at similar intensities. Indeed, this dramatic enhancement is due the non-centrosymmetric nature of AlGaAs. Moreover, in contrast to plasmonics, resonances of high-index dielectric nanoparticles provide a mode volume that is not limited to interfaces and thus lead to greater nonlinear conversion efficiencies. Several strategies have been employed to enhance harmonic nonlinearities including dielectric nanopillars in a dimer configuration [9] and a metal-dielectric hybrid nanoantenna configuration [10,11] exploiting the anapole condition. Promising studies on enhanced second harmonic generation (SHG) at the nanoscale have also been performed on perovskite nanoparticles [12]. However, the generated nonlinear signal tends to remain more confined in the substrate since it typically has a greater refractive index than the surrounding air. Recently several authors have discussed some possible strategies to engineer the radiation pattern of nonlinear nano-antennas, using the properties of the fundamental frequency field (i.e., polarization state and propagation direction) as tuning parameters [13-15]. However, an unexplored possibility to optimize the nonlinear radiation pattern is the use of epsilon near zero (ENZ) substrates.

ENZ materials have attracted lots of attention because of their peculiar linear properties, such as their ability to realize perfect electromagnetic tunneling $[16,17]$ and to re-direct antennas electromagnetic radiation $[18,19]$. In particular, it has already been demonstrated that when an antenna is placed on top of an extremely low-permittivity substrate, the air is the "denser" medium with 
higher relative permittivity and therefore the antenna pattern will be predominantly backscattered into the air $[20,21]$. Their ability to be good reflectors and, at the same time, provide high electric field values, makes them also good candidates for energy harvesting purposes [22] and photocatalysis [23]. ENZ conditions, which occur when the real part of the dielectric permittivity crosses the zero, may be found in natural (e.g., low loss noble metals, some semiconductors and some polar dielectrics) and artificial materials (metamaterials) alike, where the latter are more flexible both in terms of the zero-crossing wavelength and effective losses [24-27]. Another possible path to tune the ENZ wavelength is the use of oxide materials, such as Indium Tin Oxide (ITO), Aluminum Zinc Oxide (AZO) or Dysprosium-doped Cadmium Oxide (Dy:CdO) [28-30], whose doping determines the spectral position of the ENZ crossing point. ENZ materials have been also shown to be good platforms for nonlinear processes like harmonic generation, optical bistability and soliton excitation [31-37]. In this framework, ENZ substrates have been also shown to enhance harmonic generation processes when the ENZ mode is coupled with localized surface plasmon resonances [38]. Moreover, a new approach to engineer an optical medium with an unprecedentedly large intensity-dependent refractive index using nanoantennas coupled to a thin ENZ material has been reported [39].

Here we demonstrate how one can exploit the peculiar ability of ENZ substrates to re-direct the electromagnetic radiation to increase the efficiency of nonlinear processes generated in AlGaAs nanoantennas. More specifically, we will focus on Second Harmonic (SH) generation and show that the presence of the ENZ can improve the scattered SH signal up to three orders of magnitude with respect to a dielectric substrate and up to two orders of magnitude with respect to a metallic one. This dramatic enhancement benefits a novel approach to improve nonlinear processes at the nanoscale.

\section{Results and Discussion}

We considered a nanocylinder with a height of $400 \mathrm{~nm}$ made of $\mathrm{Al}_{0.18} \mathrm{Ga}_{0.82} \mathrm{As}$ placed over a semi-infinite substrate layer. The excitation is assumed to be a $x$-polarized plane wave (see Figure 1 ) at a fundamental wavelength (FW) of $1550 \mathrm{~nm}$ with incident amplitude $\mathrm{E}_{0}$ corresponding to a pump intensity of $1.6 \mathrm{GW} / \mathrm{cm}^{2}$. A sketch of the proposed structure is reported in Figure 1a. The considered nanoantenna height is compatible with other AlGaAs structures $[6,9,13]$ already fabricated and optimized for the efficient generation of the second harmonic signal.

$\mathrm{Al}_{x} \mathrm{Ga}_{1-x} \mathrm{As}$ is a III-V semiconductor which is gaining more and more popularity for nonlinear photonics due to its non-centrosymmetric structure. Moreover, it has a large band gap enabling two photons absorption-free operation at $1.55 \mu \mathrm{m}$, a high non-resonant quadratic susceptibility $\left(d_{14} \approx 100 \mathrm{pm} / \mathrm{V}\right.$ for GaAs in the near infrared) [40], and a broad spectral window of transparency in the mid-infrared (up to $17 \mu \mathrm{m}$ ), which allows for the generation of intense second-order nonlinear optical effects [41,42]. For the dispersion of the refractive index of AlGaAs, we use the analytical model proposed in Reference [43]. At the $\mathrm{FW}$ it has a refractive index $\mathrm{n}_{\mathrm{Al} 10.18 \mathrm{Ga} 0.82 \mathrm{As}}$ equal to 3.275 that is $\operatorname{Re}(\varepsilon)=10.729, \operatorname{Im}(\varepsilon)=0$, see Table 1 .

Table 1. Summary of the permittivities of the materials used in this work.

\begin{tabular}{ccc}
\hline \multirow{2}{*}{ Material } & $\begin{array}{c}\operatorname{Re}(\varepsilon)+\mathrm{j} \operatorname{Im}(\varepsilon) \\
\mathbf{F W}\end{array}$ & $\begin{array}{c}\boldsymbol{\operatorname { R e }}(\varepsilon)+\mathrm{j} \operatorname{Im}(\varepsilon) \\
\mathbf{S H}\end{array}$ \\
\hline ENZ & $-16.48+\mathrm{j} 0.4217$ & $0.0006+\mathrm{j} 0.05$ \\
Gold & $-115.13+\mathrm{j} 11.259$ & $-22.068+\mathrm{j} 1.3708$ \\
Dielectric & $2.56+\mathrm{j} 0$ & $2.56+\mathrm{j} 0$ \\
AlGaAs & $10.729+\mathrm{j} 0$ & $12.583+\mathrm{j} 0.086$ \\
\hline
\end{tabular}

In the following we considered three different substrates where the AlGaAs nanoantenna was placed on an ENZ, a dielectric and a gold substrate. 
For the dispersion of the ENZ substrate we considered a material modelled with a single Lorentz oscillator:

$$
\varepsilon_{E N Z}(\omega)=\varepsilon_{\infty}-\frac{\omega_{p}^{2}}{\omega^{2}+i \omega \gamma}
$$

with parameters $\varepsilon_{\infty}=5.5, \omega_{p}=5.7535 \times 10^{15} \mathrm{rad} / \mathrm{s}$ and $\gamma=2.3327 \times 10^{13} \mathrm{rad} / \mathrm{s}$. The optimized substrate has the epsilon near zero condition around $\lambda=775 \mathrm{~nm}$, where the dielectric permittivity assumes a value $\varepsilon=0.0006+i 0.05$. The dielectric substrate had instead a refractive index $n_{s}$ fixed to 1.6 that is $\operatorname{Re}(\varepsilon)=2.56, \operatorname{Im}(\varepsilon)=0$ as listed in Table 1. These values correspond to those of $\mathrm{AlO}_{\mathrm{x}}$ [6]. They were chosen because of the realization that $\mathrm{AlGaAs}$ nanostructures on an $\mathrm{AlO}_{\mathrm{x}}$ layer are easily obtained in modern manufacturing processes [6]. The optical properties of gold are obtained by the Johnson and Christy interpolation [44]. For completeness, Table 1 summarizes the permittivities of the materials used in this study both at the Fundamental Wavelength (FW) and at Second Harmonic (SH) wavelength.

The experimental realization of such kind of structure will probably face various problems. However, a novel procedure for fabricating nanodisks from III-V nanowires has been recently proposed in [42]. The main advantage of this new approach of slicing nanowires with Focused Ion Beam (FIB) milling is the possibility to create disk-based configurations on any substrate. In fact, the method of FIB nanowire slicing permits one to easily create nanoantenna chains of different designs without relying on the substrate material and to make them compatible with Complementary Metal-Oxide Semiconductor (CMOS) technologies. Moreover, the proposed ENZ substrate could be realized by using transparent conductive oxide, such as ITO, GzO, AZO or doped-CdO, where the plasma frequency can be tuned by varying the doping of these materials.

We performed our simulation by means of a commercial software based on the finite element method (COMSOL Multiphysics®v. 5.2.-www.comsol.com.-COMSOL AB, Stockholm, Sweden). The model geometry is shown in Figure $1 \mathrm{~b}$. The mesh was customized to be more detailed in the cylindrical nanoantenna and in the air-substrate interface close to the nanodisk. Doing so enabled us to focus on the key phenomena of the proposed structure. The minimum mesh element size was less than $1 \mathrm{~nm}$. The domain was truncated by a perfectly matched layer (PML), and far-field calculations were done on the inner boundary of the PML domain. Surface A was used to calculate total scattered power. We evaluated three different scenarios. First, we considered the case of an ENZ substrate. We investigated the optical scattering of the AlGaAs nanodisk by changing its radius between $200 \mathrm{~nm}$ and $290 \mathrm{~nm}$. The maximum scattered power was reached for a nanocylinder with a radius of $r=232 \mathrm{~nm}$, as shown in Figure 1c.

Figure 2a shows the electric field distribution in the case of $r=232 \mathrm{~nm}$, when the substrate was the ENZ material. At $1550 \mathrm{~nm}$, the ENZ material had a real part of the permittivity equal to -16.48 and an imaginary part equal to 0.4217 (see Table 1). It can be seen how the electric field inside the cylinder reached an enhancement factor $|\mathrm{E}| /\left|\mathrm{E}_{0}\right|$ equal to 35. The high enhancement of the field at the interface between the substrate and the cylinder can be attributed to the excitation of surface plasmon polaritons of the underlying ENZ material by the dielectric nanoparticle on top [45].

Different is the case of the dielectric substrate. In the latter circumstance, the field circulated inside the disk (see Figure 2 b) because a low refractive index $\left(n_{s} \approx 1.6\right)$, enabling sub-wavelength optical confinement in the nanocavity by total internal reflection. The multipole analysis deduced from the distributions of the induced electric current, density had been carried out [46]. For $r=232 \mathrm{~nm}$ the scattering behaviour was dominated by the electric and magnetic dipolar contributions as can be also concluded by looking at the field distribution. Finally, the case of gold substrate was considered. See Table 1 for the gold permittivity at the FW. The mode excited inside the nanoantenna resembled the one of the ENZ substrate but the field enhancement was more than 8 times lower. As the real part of the permittivity of the substrate became more negative, the electric field generated by the antenna was reduced because the substrate behaves as an electric conductor, cancelling the dipolar moment at the direct surface by an imaginary mirror dipole in the substrate. This is similar to the case of 
increasing the imaginary part of the permittivity [47]. We stress that, even though the radius of the cylinder was optimized for the ENZ substrate, no significant field enhancement was obtained for the other substrates using a different radius. We then simulated the second harmonic generation from the proposed structure. Since AlGaAs is a non-centrosymmetric material, we had a strong volume contribution which could enhance the SH signal.

(a)

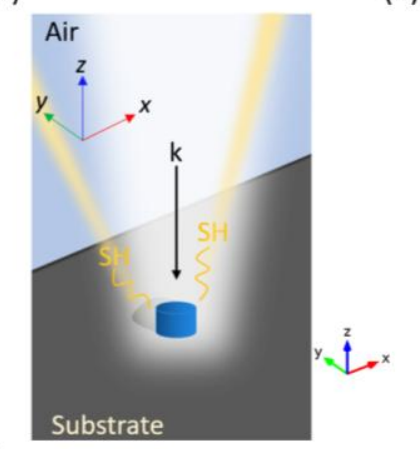

(c) 40

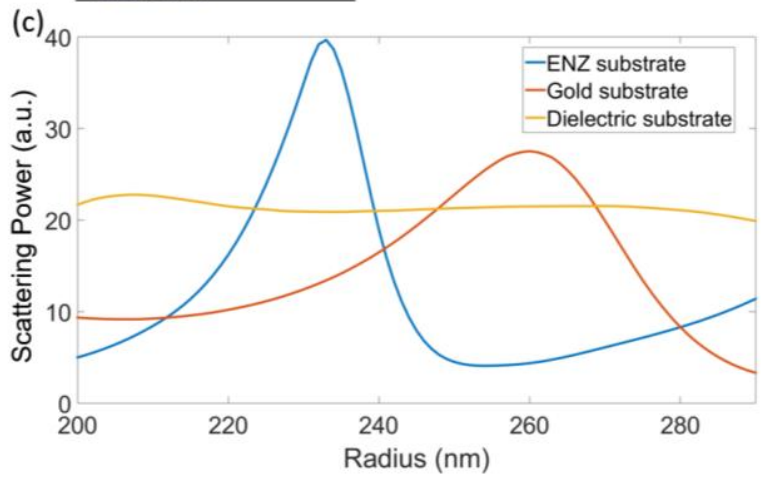

(b)

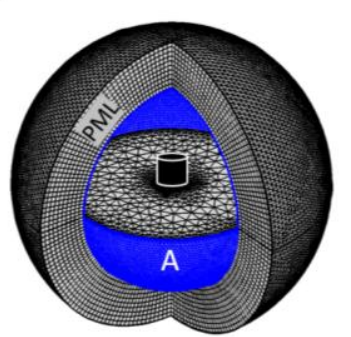

Figure 1. (a) Sketch of the proposed structure; (b) simulated model with the discretization mesh; (c) scattering power at varying cylinder radius $(h=400 \mathrm{~nm})$ for the different considered substrates, $I_{0}=1.6 \mathrm{GW} / \mathrm{cm}^{2}$.

We first note that, among the different resonant modes that were observed in the nanodisks in Figure 1c, the resonance at $r=232 \mathrm{~nm}$ in the case of ENZ substrate, showed the strongest scattering power and electric field enhancement inside the structure. This can potentially enhance the nonlinear optical response achievable from these nanoantennas. We recall that the second harmonic (SH) wavelength falls at the ENZ condition wavelength of the optimized substrate. The second-order nonlinear susceptibility tensor of (100)-grown AlGaAs is anisotropic and has only off-diagonal elements $\chi_{i j k}^{(2)}$ with $i \neq j \neq k$. In the calculations, we used $\chi^{(2)}=100 \mathrm{pm} / \mathrm{V}$ as reported for $\mathrm{Al}_{0.18} \mathrm{Ga}_{0.82}$ as in [48]. We thus solved the problem of the SH wavelength using the calculated fields at the FW to define the second harmonic source as external current density. The $i$-th component of the external current density $J_{i}$ is expressed as:

$$
J_{i}=j \omega_{S H} \varepsilon_{0} \chi^{(2)} E_{F F, j} E_{F F, k}
$$

where $\varepsilon_{0}$ is the vacuum permittivity and $E_{F F, i}$ is the $i$-th component of the electric field at the FW. Note that here we are assuming that no $\mathrm{SH}$ signal is coming from the substrate.

Figure $2 \mathrm{~d}-\mathrm{f}$ shows the electric field distribution at the Second Harmonic wavelength $\mathrm{SH}$. Figure 2a,d suggest that the use of the ENZ enlarged the mode-matching between the mode at FW and SH wavelengths. The overlap integral estimation [5] clarified that the maximum overlap (about $46 \%$ ) was reached for the ENZ substrate whereas was only about $42 \%$ for the gold substrate and $39 \%$ for the dielectric substrate. 

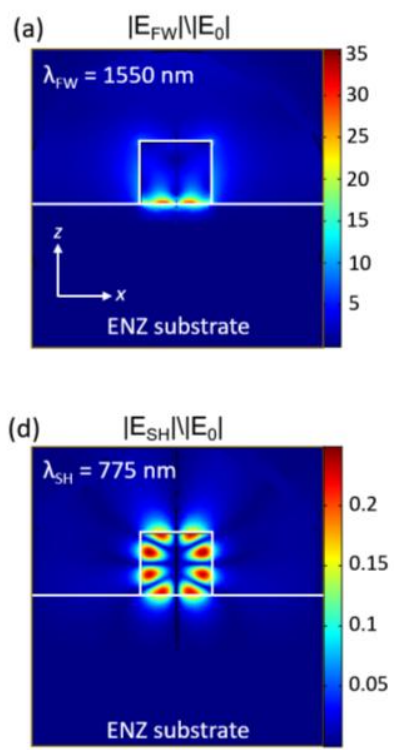
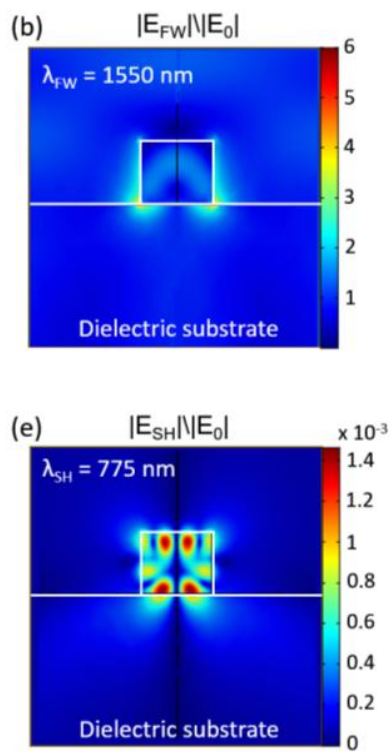
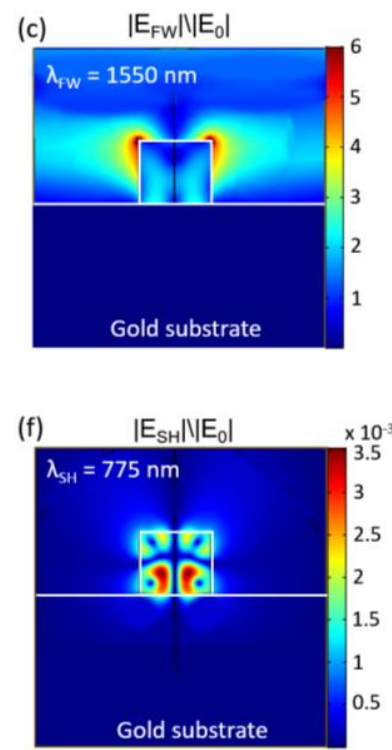

Figure 2. Electric field enhancement at the fundamental wavelength $\mathrm{FW}$ (second harmonic $\mathrm{SH}$ ) in the case of nanocylinder of radius $232 \mathrm{~nm}$, and height $h$ equal to $400 \mathrm{~nm}$ placed over the ENZ ((a) at FW and (d) at $\mathrm{SH})$, the dielectric $((\mathbf{b})$ at $\mathrm{FW}(\mathbf{e})$ at $\mathrm{SH})$ and the gold substrate $((\mathbf{c})$ at FW and (f) at $\mathrm{SH})$.

The geometrical conversion efficiency is defined as:

$$
\eta_{S H G}=\frac{\int_{A} \vec{S}_{S H} \cdot \hat{n} d a}{I_{0} \times \pi r^{2}}
$$

where $\vec{S}_{S H}$ is the Poynting vector of the SH field, $\hat{n}$ is the unit vector normal to a surface A enclosing the antenna, and $I_{0}$ is the incident field intensity.

We numerically found a SH conversion efficiency of up to $3 \times 10^{-3}$ in the case of nanodisks placed over the ENZ substrate. We also evaluated how much SH power was redirected in the air region by defining $\Gamma$ as the ratio between the $\mathrm{SH}$ power radiated in the air and the $\mathrm{SH}$ power radiated in the substrate region through surface A enclosing the nanodisk (see Figure 1b). For the ENZ substrate, $\Gamma$ reached the maximum value of 1191 . This means that in the proposed structure we were able to obtain a very high SH efficiency and at the same time, the second harmonic signal was redirected into the upper region where the air was present. On the other hand, in the case of the dielectric substrate, $\Gamma$ is equal to 0.28 meaning that most of the $\mathrm{SH}$ signal was redirected into the substrate, as shown in Figure $3 \mathrm{~b}$, and the SHG efficiency was about $4 \times 10^{-7}$. A good SH signal redirection was instead obtained in the case of a gold substrate, where $\Gamma$ was equal to 836 , see Figure $3 \mathrm{c}$. However, the achieved efficiency did not exceed $8 \times 10^{-7}$. In this case, the SH efficiency limiting factor was represented by the poor electric field enhancement inside the cylinder at the fundamental wavelength. The second harmonic fields in Figure 2d-f indicate that higher order modes were excited inside the structure at the SH wavelength. Such higher order multipoles were responsible for the obtained far field.

We stress that the analysis performed did not take surface SHG effects into account, but we only considered the quadratic bulk nonlinearity contribution since the latter term has been shown to be dominant in these kinds of dielectric structures [5-9]. A complete analysis and comparison between surface and volume nonlinear contributions was postponed to a future effort. We note that, although all the calculations have been performed for a cylinder optimized on the ENZ substrate, even if we consider the other substrates with the respective optimized cylinder radius, we obtained a maximum $\mathrm{SH}$ conversion efficiency of less than $10^{-6}$ for the $\mathrm{AlO}_{\mathrm{x}}$ substrate $(r=205 \mathrm{~nm})$ and $5.5 \times 10^{-5}$ for the gold substrate $(r=260 \mathrm{~nm})$. 
(a)

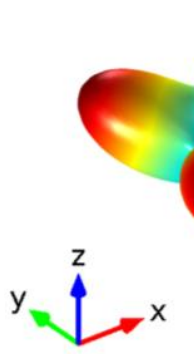

(b)

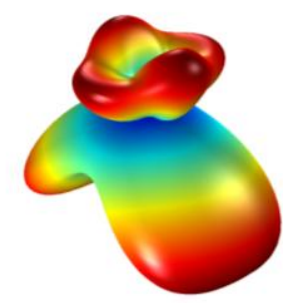

(c)

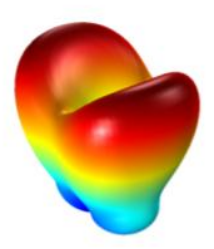

Figure 3. SH Far Field in the case of AlGaAs cylinder ( $r=232 \mathrm{~nm}, h=400 \mathrm{~nm}$ ) placed over (a) the ENZ, (b) the dielectric and (c) the gold substrate when excited by a plane wave.

Moreover, by using the eigenfrequency analysis in COMSOL Multiphysics, one can estimate the $Q$ factor of the mode for the proposed structure in the different scenarios. The linear scattering power spectra for the different substrates are reported in Figure 4a, for a nanodisk with $r=232 \mathrm{~nm}$ and $h=400 \mathrm{~nm}$. For the ENZ substrate, the scattering spectra showed a Fano-like behavior. A Fano resonance originates from the coupling between a broad "bright" mode resonator, that is accessible from free space, and a narrow "dark" mode resonator which is less-accessible, or inaccessible, from free-space. When the two resonances are brought in close proximity in both the spatial and frequency domains, a coupling is possible. This leads to a total resonance in the system, coming from the interference between the two pathways. We could attribuite this Fano resonance to the excitation of the broad magnetic dipolar resonance in the AlGaAs nanodisk that interfered with the SPP excited at the interface between the ENZ material and the dielectric resonator. The mode of the ENZ substrate (Figure 4b) has a $Q$ factor of 25 for a pump wavelength of $1550 \mathrm{~nm}$. If the cylinder is slightly moved upwards from the substrate one can find a field distribution as shown in Figure 4c with $Q$ factor that decreases by increasing the distance of the cylinder from the substrate.

In the limit case of infinite distance, the dielectric antenna can be considered as completely surrounded by air. In this latter situation, the closest mode (which is a magnetic resonance) has a $Q$ factor of 9 at the wavelength of about $1695 \mathrm{~nm}$, as shown in Figure 4d. The analysis of the nanostructure placed at different heights from the substrate demonstrates that the high enhancement of the field at the interface was due to the excitation of surface plasmon polaritons of the underlying ENZ substrate by the AlGaAs nanodisk on top. The dielectric nanoparticle helps provides the appropriate momentum ( $k$-vector) to couple light at the interface. As far as concerning the substrate with refractive index 1.6, the closest mode that one can excite is a magnetic resonance with a $Q$ factor of 6 for a wavelength equal to $1710 \mathrm{~nm}$ (Figure 4e). This is in perfect agreement with the known effect of broadening and redshifting of the resonances that are obtained when a nanostructure is placed on a dielectric substrate with respect to the case of structure in homogenous media (usually assumed to be air). Finally, when the AlGaAs nanocylinder was placed over the gold substrate, the closest mode had a $Q$ factor equal to 20 for a wavelength of $1460 \mathrm{~nm}$, see Figure $4 \mathrm{f}$.

Therefore, to conclude, the mode with the highest $Q$ factor excitable at $1550 \mathrm{~nm}$ is the one obtained in the case of ENZ substrate. This high-quality mode is supported by the nanoantenna results in the enhanced near-field distribution, as represented in Figure 2a. 
(a)

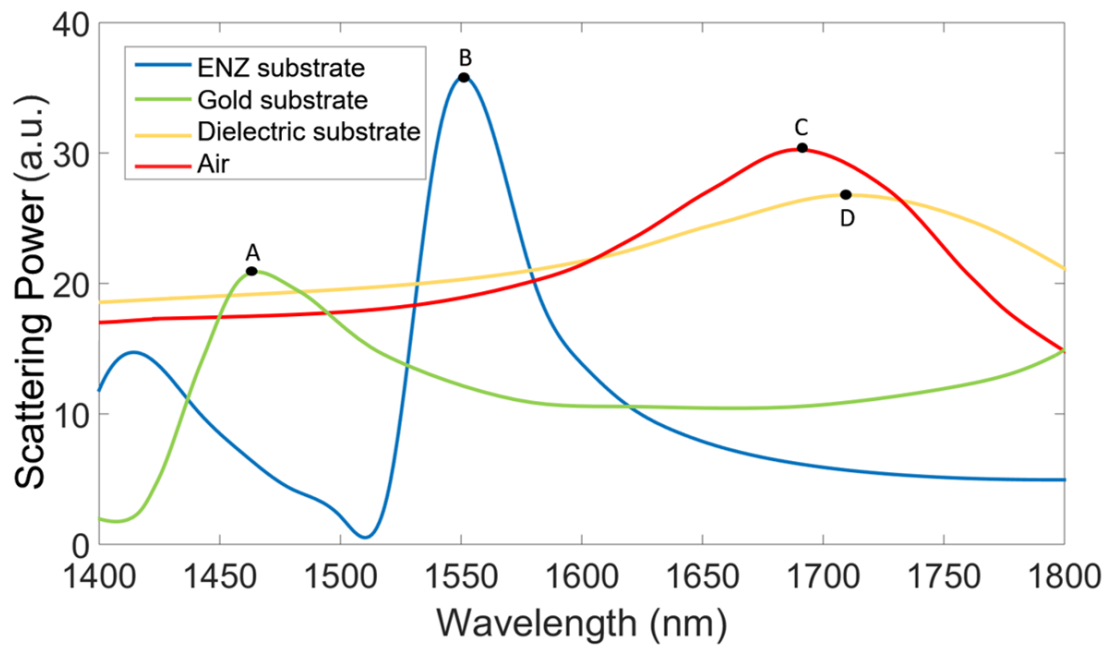

(b)

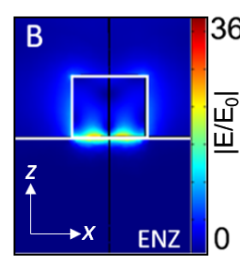

(c)

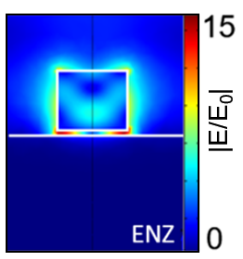

(d)

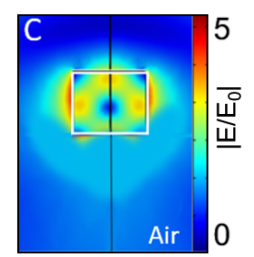

(e)

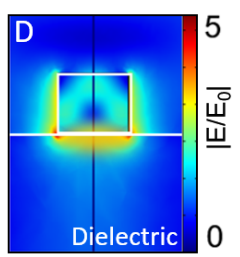

(f)

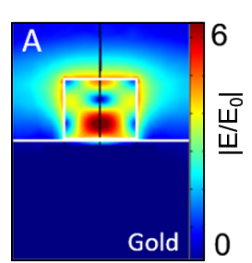

Figure 4. (a) Scattering spectra for an AlGaAs nanocylinder with $r=232 \mathrm{~nm}$ and $h=400 \mathrm{~nm}$ placed over the different substrates. (b) Near-field distributions of the electric field enhancement for nanodisk placed over the ENZ substrate at the pump wavelength of $1550 \mathrm{~nm}$ and (c) with a gap from the substrate equal to $30 \mathrm{~nm}$. (d) Near-field distribution for the same AlGaAs nanoantenna completely surrounded by air at $1694 \mathrm{~nm}$; in the case of dielectric substrate (d) at $1710 \mathrm{~nm}$ and in the case of gold substrate (e) at $1460 \mathrm{~nm}$.

\section{Conclusions}

We performed a theoretical analysis to show how to enhance the collection of the second harmonic signal generated from AlGaAs resonators. The possibility of enhancing optical nonlinear effects by introducing an epsilon-near-zero substrate has been demonstrated. More precisely, in this work we have shown that it is possible to enhance the second harmonic efficiency and directivity by exploiting simultaneously the strong electric field enhancement induced inside the structure at the FW and the ENZ condition at the $\mathrm{SH}$ wavelength. By designing a cylindrical nanoantenna optimized over an ENZ substrate we obtained a second harmonic conversion efficiency on the order of $10^{-3}$ for a pump intensity of $I_{0}=1.6 \mathrm{GW} / \mathrm{cm}^{2}$. These results offer the opportunity to re-think the design of novel nonlinear photonic metadevices, with improved capabilities for on-chip communication, information processing and sensing.

Author Contributions: Conceptualization, D.R., M.A.V., C.D.A.; Methodology, D.R., M.A.V., C.D.A.; Investigation, D.R., M.A.V., C.D.A.; Writing - original draft preparation, D.R., M.A.V., C.D.A.; Writing - review and editing, D.R., M.A.V., C.D.A.

Funding: This research was partially funded by the Rita Levi Montalcini Program and by the Erasmus Mundus NANOPHI Project-Education, Audiovisual and Culture Executive Agency (EACEA) (5659/002-001).

Conflicts of Interest: The authors declare no conflict of interest.

\section{References}

1. Kuznetsov, A.I.; Miroshnichenko, A.E.; Brongersma, M.L.; Kivshar, Y.S.; Lukyanchuk, B. Optically resonant dielectric nanostructures. Science 2016, 354, 6314. [CrossRef] [PubMed]

2. Smirnova, D.; Kivshar, Y.S. Multipolar nonlinear nanophotonics. Optica 2016, 3, 1241-1255. [CrossRef] 
3. Decker, M.; Staude, I. Resonant dielectric nanostructures: A low-loss platform for functional nanophotonics. J. Opt. 2016, 18, 103001. [CrossRef]

4. Rocco, D.; Carletti, L.; Locatelli, A.; De Angelis, C. Controlling the directivity of all-dielectric nanoantennas excited by integrated quantum emitters. J. Opt. Soc. Am. B 2017, 34, 1918-1922. [CrossRef]

5. Carletti, L.; Locatelli, A.; Stepanenko, O.; Leo, G.; De Angelis, C. Enhanced second-harmonic generation from magnetic resonance in AlGaAs nanoantennas. Opt. Express 2015, 23, 26544-26550. [CrossRef] [PubMed]

6. Gili, V.F.; Carletti, L.; Locatelli, A.; Rocco, D.; Finazzi, M.; Ghirardini, L.; Favero, I.; Gomez, C.; Lemaître, A.; Celebrano, M.; De Angelis, C.; et al. Monolithic AlGaAs second-harmonic nanoantennas. Opt. Express 2016, 24, 15965-15971. [CrossRef] [PubMed]

7. Camacho-Morales, R.; Rahmani, M.; Kruk, S.; Wang, L.; Xu, L.; Smirnova, D.A.; Solntsev, A.S.; Miroshnichenko, A.; Tan, H.H.; Karouta, F.; et al. Nonlinear Generation of Vector Beams From AlGaAs Nanoantennas. Nano Lett. 2016, 16, 7191-7197. [CrossRef] [PubMed]

8. Liu, S.; Sinclair, M.B.; Saravi, S.; Keeler, G.A.; Yang, Y.; Reno, J.; Peake, G.M.; Setzpfandt, F.; Staude, I.; Pertsch, T; et al. Resonantly Enhanced Second-Harmonic Generation Using III-V Semiconductor All-Dielectric Metasurfaces. Nano Lett. 2016, 16, 5426-5432. [CrossRef] [PubMed]

9. Rocco, D.; Gili, V.F.; Ghirardini, L.; Carletti, L.; Favero, I.; Locatelli, A.; Marino, G.; Neshev, D.N.; Celebrano, M.; Finazzi, M.; et al. Tuning the second-harmonic generation in AlGaAs nanodimers via non-radiative state optimization. Photonics Res. 2018, 6, B6-B12. [CrossRef]

10. Grinbalt, G.; Li, Y.; Nielsen, M.P.; Oulton, R.F.; Maier, S.A. Enhanced third harmonic generation in single germanium nanodisks excited at the anapole mode. Nano Lett. 2016, 16, 4635-4640. [CrossRef] [PubMed]

11. Gili, V.F.; Ghirardini, L.; Rocco, D.; Marino, G.; Favero, I.; Roland, I.; Pellegrini, G.; Duò, L.; Finazzi, M.; Carletti, L.; et al. Metal-dielectric hybrid nanoantennas for efficient frequency conversion at the anapole mode. Beilstein J. Nanotechnol. 2018, 9, 2306-2314. [CrossRef] [PubMed]

12. Timpu, F.; Sergeyev, A.; Hendricks, N.R.; Grange, R. Second-harmonic enhancement with Mie resonances in perovskite nanoparticles. ACS Photonics 2016, 4, 76-84. [CrossRef]

13. Carletti, L.; Rocco, D.; Locatelli, A.; De Angelis, C.; Gili, V.F.; Ravaro, M.; Favero, I.; Leo, G.; Finazzi, M.; Ghirardini, L.; et al. Controlling second-harmonic generation at the nanoscale with monolithic AlGaAs-on-AlO ${ }_{x}$ antennas. Nanotechnology 2017, 28, 114005. [CrossRef] [PubMed]

14. Kruk, S.S.; Camacho-Morales, R.; Xu, L.; Rahmani, M.; Smirnova, D.A.; Wang, L.; Tan, H.H.; Jagadish, C.; Neshev, D.N.; Kivshar, Y.S. Nonlinear Optical Magnetism Revealed by Second-Harmonic Generation in Nanoantennas. Nano Lett. 2017, 6, 3914-3918. [CrossRef] [PubMed]

15. Guasoni, M.; Carletti, L.; Neshev, D.; De Angelis, C. Theoretical Model for Pattern Engineering of Harmonic Generation in All-Dielectric Nanoantennas. IEEE J. Quantum Electron. 2017, 53, 1-5. [CrossRef]

16. Alù, A.; Silveirinha, M.G.; Salandrino, A.; Engheta, N. Epsilon-near-zero metamaterials and electromagnetic sources: Tailoring the radiation phase pattern. Phys. Rev. B 2007, 75, 155410. [CrossRef]

17. Silveirinha, M.; Engheta, N. Tunneling of Electromagnetic Energy through Subwavelength Channels and Bends using E-Near-Zero Materials. Phys. Rev. Lett. 2006, 97, 157403. [CrossRef] [PubMed]

18. Enoch, S.; Tayeb, G.; Sabouroux, P.; Guérin, N.; Vincent, P. A Metamaterial for Directive Emission. Phys. Rev. Lett. 2002, 89, 213902. [CrossRef] [PubMed]

19. Lovat, G.; Burghignoli, P.; Capolino, F.; Jackson, D.R.; Wilton, D.R. Analysis of directive radiation from a line source in a metamaterial slab with low permittivity. IEE Trans. Antennas Propag. 2006, 54, 1017-1030. [CrossRef]

20. Memarian, M.; Eleftheriades, G.V. Dipole radiation near anisotropic low-permittivity media. Prog. Electromagn. Res. 2013, 142, 437-462. [CrossRef]

21. Guclu, C.; Campione, S.; Capolino, F. Array of dipoles near a hyperbolic metamaterial: Evanescent-topropagating Floquet wave transformation. Phys. Rev. B 2014, 89, 155128. [CrossRef]

22. Labelle, A.J.; Bonifazi, M.; Tian, Y.; Wong, C.; Hoogland, S.; Favraud, G.; Walters, G.; Sutherland, B.; Liu, M.; Li, J.; et al. Broadband Epsilon-near-Zero Reflectors Enhance the Quantum Efficiency of Thin Solar Cells at Visible and Infrared Wavelengths. ACS Appl. Mater. Interfaces 2017, 9, 5556-5565. [CrossRef] [PubMed]

23. Tian, Y.; García de Arquer, F.P.; Dinh, C.-T.; Favraud, G.; Bonifazi, M.; Li, J.; Liu, M.; Zhang, X.; Zheng, X.; Kibria, M.G.; et al. Enhanced Solar-to-Hydrogen Generation with Broadband Epsilon-Near-Zero Nanostructured Photocatalysts. Adv. Mater. 2017, 29, 1701165. [CrossRef] [PubMed] 
24. Campione, S.; Albani, M.; Capolino, F. Complex modes and near-zero permittivity in 3D arrays of plasmonic nanoshells: Loss compensation using gain. Opt. Mater. Express 2011, 1, 1077-1089. [CrossRef]

25. Campione, S.; Capolino, F. Composite material made of plasmonic nanoshells with quantum dot cores: Loss-compensation and epsilon-near-zero physical properties. Nanotechology 2012, 23, 235703. [CrossRef] [PubMed]

26. Ciattoni, A.; Marinelli, R.; Rizza, C.; Palange, E. Epsilon-Near-zero materials in the near-infrared. Appl. Phys. B 2013, 110, 23-26. [CrossRef]

27. Xiao, S.; Drachev, V.P.; Kildishev, A.V.; Ni, X.; Chettiar, U.K.; Yuan, H.-K.; Shalaev, V.M. Loss-free and active optical negative-index metamaterials. Nature 2010, 466, 735-738. [CrossRef] [PubMed]

28. Naik, G.V.; Kim, J.; Boltasseva, A. Oxides and nitrides as alternative plasmonic materials in the optical range. Opt. Mater. Express 2011, 1, 1090-1099. [CrossRef]

29. Naik, G.V.; Liu, J.; Kildishev, A.V.; Shalaev, V.M.; Boltasseva, A. Demonstration of Al:ZnO as a plasmonic component for near-infrared metamaterials. Proc. Natl. Acad. Sci. USA 2012, 109, 8834-8838. [CrossRef] [PubMed]

30. Sachet, E.; Shelton, C.T.; Harris, J.S.; Gaddy, B.E.; Irving, D.L.; Curtarolo, S.; Donovan, B.F.; Hopkins, P.E.; Sharma, P.A.; Sharma, A.L.; et al. Dysprosium-doped cadmium oxide as a gateway material for mid-infrared plasmonics. Nat. Mater. 2015, 14, 414-420. [CrossRef] [PubMed]

31. Vincenti, M.A.; de Ceglia, D.; Ciattoni, A.; Scalora, M. Singularity-driven second- and third-harmonic generation at $\varepsilon$-near-zero crossing points. Phys. Rev. A 2011, 84, 063826. [CrossRef]

32. Ciattoni, A.; Spinozzi, E. Efficient second-harmonic generation in micrometer-thick slabs with indefinite permittivity. Phys. Rev. A 2012, 85, 043806. [CrossRef]

33. Vincenti, M.A.; Campione, S.; de Ceglia, D.; Capolino, F.; Scalora, M. Gain-assisted harmonic generation in near-zero permittivity metamaterials made of plasmonic nanoshells. New J. Phys. 2012, 14, 103016. [CrossRef]

34. Ciattoni, A.; Rizza, C.; Palange, E. Extreme nonlinear electrodynamics in metamaterials with very small linear dielectric permittivity. Phys. Rev. A 2010, 81, 043839. [CrossRef]

35. De Ceglia, D.; Campione, S.; Vincenti, M.A.; Capolino, F.; Scalora, M. Low-damping epsilon-near-zero slabs: Nonlinear and nonlocal optical properties. Phys. Rev. B 2013, 87, 155140. [CrossRef]

36. Rizza, C.; Ciattoni, A.; Palange, E. Two-peaked and flat-top perfect bright solitons in nonlinear metamaterials with epsilon near zero. Phys. Rev. A 2011, 83, 053805. [CrossRef]

37. Vincenti, M.A.; Kamandi, M.; de Ceglia, D.; Guclu, C.; Scalora, M.; Capolino, F. Second-harmonic generation in longitudinal epsilon-near-zero materials. Phys. Rev. B 2017, 96, 045438. [CrossRef]

38. Wen, X.; Li, G.; Gu, C.; Zhao, J.; Wang, S.; Jiang, C.; Palomba, S.; de Sterke, C.M.; Xiong, Q. Doubly Enhanced Second Harmonic Generation through Structural and Epsilon-near-Zero Resonances in TiN Nanostructures. ACS Photonics 2018, 5, 2087-2093. [CrossRef]

39. Alam, M.Z.; Schulz, S.A.; Upham, J.; De Leon, I.; Boyd, R.W. Large optical nonlinearity of nanoantennas coupled to an epsilon-near-zero material. Nat. Photonics 2018, 12, 79. [CrossRef]

40. Ohashi, M.; Kondo, T.; Ito, R.; Fukatsu, S.; Shiraki, Y.; Kumata, K.; Kano, S.S. Determination of quadratic nonlinear optical coefficient of $\mathrm{Al}_{x} \mathrm{Ga}_{1-x}$ As system by the method of reflected second harmonics. J. Appl. Phys. 1993, 74, 596-601. [CrossRef]

41. Vabishchevich, P.P.; Liu, S.; Sinclair, M.B.; Keeler, G.A.; Peake, G.M.; Brener, I. Enhanced second-harmonic generation using broken symmetry III-V semiconductor fano metasurfaces. ACS Photonics 2018, 5, 1685-1690. [CrossRef]

42. Timofeeva, M.; Lang, L.; Timpu, F.; Renaut, C.; Bouravleuv, A.; Shtrom, I.V.; Cirlin, G.; Grange, R. Anapoles in Free-Standing III-V Nanodisks Enhancing Second-Harmonic Generation. Nano Lett. 2018, 6, 3695-3702. [CrossRef] [PubMed]

43. Gehrsitz, S.; Reinhart, F.K.; Gourgon, C.; Herres, N.; Vonlanthen, A.; Sigg, H. The refractive index of $\mathrm{Al}_{x} \mathrm{Ga}_{1-x} \mathrm{As}$ below the band gap: Accurate determination and empirical modeling. J. Appl. Phys. 2000, 87, 7825-7837. [CrossRef]

44. Johnson, P.B.; Christy, R.W. Optical constants of the noble metals. Phys. Rev. B 1972, 6, 4370. [CrossRef]

45. Hutter, T.; Huang, F.M.; Elliot, S.R.; Mahajan, S. Near-Field plasmonics of an individual dielectric nanoparticle above a metallic substrate. J. Phys. Chem. C 2013, 117, 7784-7790. [CrossRef]

46. Grahn, P.; Shevchenko, A.; Kaivola, M. Electromagnetic multipole theory for optical nanomaterials. New J. Phys. 2012, 14, 093033. [CrossRef] 
47. Kim, J.; Dutta, A.; Naik, G.V.; Giles, A.J.; Bezares, F.J.; Ellis, C.T.; Tischler, J.G.; Mahmoud, A.M.; Caglayan, H.; Glembocki, O.J.; et al. Role of epsilon-near-zero substrates in the optical response of plasmonic antennas. Optica 2016, 3, 339-346. [CrossRef]

48. Yang, Z.; Chak, P.; Bristow, A.D.; van Driel, H.M.; Iyer, R.; Aitchison, J.S.; Smirl, A.L.; Sipe, J.E. Enhanced second-harmonic generation in AlGaAs microring resonators. Opt. Lett. 2007, 32, 826-828. [CrossRef] [PubMed]

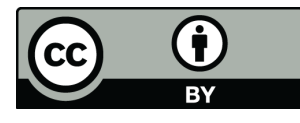

(C) 2018 by the authors. Licensee MDPI, Basel, Switzerland. This article is an open access article distributed under the terms and conditions of the Creative Commons Attribution (CC BY) license (http:/ / creativecommons.org/licenses/by/4.0/). 